\title{
Liver Transplantation: What Every Gastroenterologist Should Know about It
}

\author{
Reza F. Saidi ${ }^{1}$
}

1. Department of Surgery, Digestive Disease Research Institute, Shariati Hospital, Tehran University of Medical Sciences, Tehran, Iran

* Corresponding Author: Reza F. Saidi, MD, FICS, FACS Department of Surgery, Shariati Hospital, Digestive Disease Research Institute, Tehran University of Medical Sciences, Tehran, Iran

Tel: + 982182415104

Fax: + 982188633039

Email: srsaidi@tums.ac.ir

Received: 11 Dec. 2018

Accepted: 14 Mar. 2018

\section{ABSTRACT}

Liver Transplantation (LT) is the treatment of choice for patients with end-stage liver disease. Improvement in outcomes (allograft and patient survival) has led to widespread use of LT worldwide. This success is due to improvement in patient selection, transplantation surgery, anesthesia/postoperative care, and immunosuppression management. This review will focus on different aspects of LT, which every physician should know to provide better patient care.

\section{KEYWORDS:}

Liver Transplantation, Allograft, Immunosuppression

Please cite this paper as:

Saidi RF, Liver Transplantation: What Every Gastroenterologist Should Know about It. Middle East J Dig Dis 2018;10:69-74. doi: 10.15171/mejdd.2018.93.

\section{INTRODUCTION}

Liver Transplantation (LT) is a lifesaving procedure and the treatment of choice for patients suffering from end-stage liver disease (ESLD). The first successful LT in Iran was performed at Shiraz University of Medical Sciences in $1992 .{ }^{1}$ Since then, several LT programs were developed throughout the country. ${ }^{2,3}$ This review will discuss different aspects of LT, which every gastroenterologist should know.

\section{Chronic Liver Disease in Iran}

Chronic liver diseases including liver cancer are presently the 5th etiology of mortality in Iran with more than 8000 death per year. ${ }^{4,5}$ The most common cause of ESLD was HBV-related cirrhosis until 2005. ${ }^{4}$ Following universal neonatal HBV vaccination in 1992 and adulthood vaccination in 2005, the incidence of HBV infection in Iran declined dramatically. The most common causes of ESLD are mainly steatohepatitis in men and autoimmune hepatitis in women. ${ }^{5,6}$ Cholestatic liver disease especially primary sclerosing cholangitis is also becoming common due to the emergence of inflammatory bowel disease (IBD) epidemic in Iran. ${ }^{7,8}$ Hepatitis $\mathrm{C}$ and alcoholic cirrhosis are not common. Plan for hepatitis $\mathrm{C}$ virus (HCV) eradication with very effective new generation of anti-HCV medication would make the need for LT secondary to $\mathrm{HCV}$ much less common in near future. ${ }^{9}$ Non-alcoholic fatty liver disease (NAFLD) is also on the rise in Iran. ${ }^{9}$ ESLD secondary to viral, and autoimmune etiologies, and NAFLD constitute the etiology of up to $50 \%$ of admissions and mortalities at a major academic center 
Table 1: Indications (A) and contraindications (B) of liver transplantation

\begin{tabular}{l}
\hline A. Indications \\
\hline 1- Alcoholic cirrhosis \\
2- Viral cirrhosis: Hepatitis C, Hepatitis B \\
3- Cholestatic liver disease: PSC, PBC \\
4- Metabolic disorders: alpha-1 antitrypsin deficiency, Wilson disease, hemochromatosis, familiar amyloidotic polyneuropathy \\
5- Acute liver failure \\
6- Hepatic neoplasms: hepatocellular carcinoma, hemangioepithelioma, hilar cholangiocarcinoma, metastatic neuroendocrine tumors \\
7- others: severe liver trauma, Budd-Chiari syndrome \\
\hline B. Contraindications \\
\hline 1- Lack of patient compliance \\
2- Poor psychological support \\
3- Absence of sobriety \\
4- Active drug abuse \\
5- Advanced cardiovascular or pulmonary diseases \\
6- Uncontrolled sepsis or multiple organ failure \\
7- AIDS \\
8- Active cancer (except selected cases of Hepatocellular Carcinoma \\
\hline
\end{tabular}

in Iran for both sexes. ${ }^{10}$

Malekzadeh ${ }^{6}$ and her colleagues studied more than 120,000 deaths in Iran in 2010. Among adults aged 15 to 49 years, the main causes of death due to digestive disease were gastrointestinal (GI) and liver cancer, and cirrhosis. Their findings showed similar results even among adults older 50 years. Sepanlou and her colleagues also showed that from 1990 to 2010, there were approximately 8000 deaths annually due to cirrhosis and its complications in Iran. ${ }^{7}$ The authors estimated that there were annual 2500 deaths caused by HBV cirrhosis, 3400 deaths due to NAFLD, 1600 deaths due to HCV cirrhosis, and 500 deaths due to alcoholic liver disease in Iran. ${ }^{7}$

\section{Indications and contraindications for $\mathrm{LT}$}

Patients with ESLD should be considered for LT after extensive multidisciplinary investigations. Lack of patient compliance, poor psychological support, absence of sobriety, active drug abuse, advanced cardiovascular or pulmonary diseases, uncontrolled sepsis, irreversible multiple organ failure, AIDS, and active cancer (except selected cases of hepatocellular carcinoma [HCC]) are contraindications for LT (Table 1). HBV and cryptogenic cirrhosis (most likely due to NAFLD) are the most common indications for LT in Iran. ${ }^{3}$ Patients with compensated cirrhosis are not candidates as their survival is better without LT. Decompensation can manifest as bleeding from esophageal varies, intractable ascites, hepatic encephalopathy, hepatorenal syndrome, or hepatocellular carcinoma. ${ }^{11}$

\section{Sources of Liver Allografts}

There has been a significant progress regarding organ donation in Iran. Donors per million population (PMP) has increased to 25 in recent years. ${ }^{10}$ There have been 5000 brain death donors in Iran last year. However only 1500 cases proceeded to organ donation due to low conversion rate. There is a need to increase public awareness regarding organ donation. There is still no active deceased donor program in several providences in. ${ }^{3}$ Increase in public awareness and development of more deceased donation networks throughout the country would lead to improve public access to LT in Iran.

Most of livers are procured from brain death donors. Nevertheless, the increasing number of patients dying on the waiting list due to the shortage of liver transplants has prompted the transplant community to use more organ resources. Their effort to expand the donor pool has provided alternative ways of organ supply, including using live donors, split-LT, ${ }^{12}$ and utilization of expanded criteria donors (ECD), or marginal allografts. The ideal donor criteria include young donors died after trauma. An ECD liver might be considered but not limited to the following: donor age $>65$ years, steatosis $>$ $30 \%$ of liver allograft, peak donor serum sodium level $>155 \mathrm{mEq} / \mathrm{L}$, use of high dose or multiple 
vasopressor agents, prolonged intensive care unit stay, or prolonged cold ischemia time. ${ }^{13}$

Living-donor liver transplantation (LDLT) is an established treatment for ESLD. In Asian countries, approximately $90 \%$ of donor organs for LT are obtained from live donors, as the deceased donor rate is low due to social and religious factors. LDLT has several well documented advantages, including the use of a graft from a healthy donor with short ischemic time, the ability to schedule surgery electively, a reduced risk of the recipient dying on the waiting list, and the chance for the recipient to be medically stabilized. Disadvantages of LDLT are the higher rate of surgical complications for both the donor and recipient, and a potential risk of small-forsize syndrome. LDLT carries inherent risks for the healthy donor. ${ }^{14}$ Therefore, careful selection of the donor and recipient is crucial to minimize the risks and complications, and to obtain an acceptable outcome. Initially donors undergo psychosocial evaluation to assure there is no coercion. Next, donors are evaluated by clinical examination and serological testing for liver diseases, renal diseases, and viral hepatitis. Diagnostic studies to evaluate the vascular and biliary anatomy of the liver are done next. Several options for preoperative imaging are available, which include non-invasive modalities such as multi-phase liver computed tomography, duplex ultrasonography, and magnetic resonance imaging. The risk of donor death after partial liver donation has been reported 1:500 worldwide. The complication rate is about $30 \%$ (bleeding, bile leak, thromboembolic event, or incisional hernia), which depends on the experience of the LT team. ${ }^{14}$

\section{Liver Allocation}

Priority of organ allocation should be based on equity and justice to use valuable organ resources. LT is a lifesaving procedure. In US, liver allocation is based on patients' risk of dying while waiting on the list for LT. This risk is calculated based on MELD score. ${ }^{3}$ MELD score is based on patient's bilirubin, creatinine $(\mathrm{Cr})$, and international normalize ration (INR). MELD score can predict the chance of dying within 3 months without LT. For example
MELD of 20 have $25 \%$ chance of dying within 3 months. MELD of 40 have more than $90 \%$ chance of death. By switching to MELD for liver allocation, mortality while on the waiting list has decreased in US without change in post-LT survival. However, MELD score has its own limitations. For example due to heavy weight on Cr in MELD calculation, the number of patients with ESLD and renal failure who receive LT has increased. MELD also has put female patients in disadvantage due to lower weight and Cr. Some patients' disease severity also is not represented by MELD such as patients with primary sclerosing cholangitis (PSC) and HCC. ${ }^{11}$ Such patients have to receive MELD exception to compete with other ESLD patients for LT. Patients with acute liver failure should be transplanted as soon as possible regardless of MELD score. ${ }^{3}$

\section{Transplantation Surgery}

LT surgery has three phases:

1- Hepatectomy phase: LT is done orthotopically. During this step the whole liver has to be removed. Due to portal hypertension, this phase can be associated with bleeding. Meticulous dissection and a good anesthesia team are crucial for the management of this step.

2- Anhepatic phase: during this step, the new allograft is brought out of the cold storage and anastomosed to the patient's circulation system. The new liver is getting warm at this moment and this phase should be completed within 30-40 minutes (warm ischemic time). During this phase, the inferior vena cava (IVC) or hepatic veins anastomosis is done first. Recipient's IVC can be replaced by donor IVC (classic technique), or donor suprahepatic IVC is sewn to the cuff of hepatic veins and donor infrahepatic IVC is ligated (piggyback technique). Next, portal vein anastomosis is done in end to end fashion.

3- Reperfusion phase: The clamps will be released and liver will be reperfused. Hepatic artery and bile duct anastomoses are done in end to end fashion. As all toxins from liver and gut can be released to systemic circulation after reperfusion (ischemia reperfusion injury), this phase can also 
be associated with hemodynamic instability, which requires an experienced anesthesia team to manage the situation. ${ }^{15}$

\section{Postoperative Care and Immunosuppression}

Patients will be transferred to ICU for monitoring postoperatively. Liver allograft will be monitored clinically and based on laboratory tests. If the liver allograft functions properly, the patient would gain consciousness, with hemodynamic stability and without coagulopathy. The liver function test should be gradually normalized. Doppler ultrasonography should be done routinely to access blood flow.

Immunosuppression (IS) regimen should start immediately. Immunosuppressive therapy includes induction and maintenance therapy. The induction agents are added to the standard immunosuppressive agents to prevent or reduce the incidence of early rejection rates following LT. Induction therapy consists of anti-CD25-receptor antibodies (basiliximab, daclizumab), an anti-CD52 monoclonal antibody (alemtuzumab), or depleting polyclonal antibodies (thymoglobulin or antithymus globulin). The standard immunosuppressive regimen is a triple therapy regimen, which consists of calcineurin inhibitors (CNI; cyclosporine or tacrolimus), steroids, and mycophenolate mofetil (MMF). CNIs are the cornerstone of the immunosuppressive regimen at most liver transplantation centers. Nevertheless, treatment with CNIs is associated with adverse effects such as nephrotoxicity, neurotoxicity, hypertension, hyperkalemia, and hyperlipidemia. Corticosteroids usually have dose-dependent side effects, which include osteoporosis, diabetes, Cushing syndrome, hypertension, and hyperlipidemia. MMF can cause bone marrow or gut toxicity.

\section{Complications}

Early complications of LT are primary non-function (PNF), vascular compromise (hepatic artery or portal vein thrombosis), infectious issues (such as line infection, pneumonia, urinary tract infection, or intra-abdominal abscesses) or bile leak.

PNF usually occurs in $4-6 \%$ of cases and is presented with encephalopathy and coagulopathy. The only treatment is immediate re-transplantation. Early hepatic artery thrombosis (within 2 weeks postoperatively) also requires re-transplantation due to sepsis from biliary complications. Portal vein thrombosis can be managed with re-operation and thrombectomy. ${ }^{15}$

Late complications are biliary strictures, vascular compromise (thrombosis or stricture), opportunistic infections (viral or fungal), metabolic syndrome, immunosuppression side effects, and malignancies (skin cancer, lymphoma).

Late hepatic artery thrombosis can be asymptomatic or present itself with biliary complications such as biloma or strictures. Fortunately most of these complications can be managed by interventional radiology or endoscopic approach. Re-transplantation is rarely required. Late vascular complication requires vascular endovascular intervention .( Table 2). ${ }^{16}$

\section{Follow-up}

Initial follow-up visits include blood tests and duplex ultrasonography of the transplanted organ to monitor for patency of vasculature, rejection, and infection. If rejection is suspected, a liver biopsy should be performed. LT recipients are at higher risk than the general population for malignancy due to IS. The most common neoplasms are skin cancer and post-transplant lymphoproliferative disease (PTLD). Cardiovascular, infectious, and malignant diseases are the most common causes of patients' death over the long term. The patients should be monitored and treated for metabolic syndrome, diabetes mellitus (DM), hyperlipidemia, and osteoporosis. They should also be followed up regarding the recurrence of the primary disease (hepatitis, NAFLD, PSC) in the allograft. Treatment should be started soon. Fortunately, the risk of allograft failure and need for re-transplantation is low.

\section{Outcome}

Several factors are predictors of post-transplantation outcomes. These factors can be divided to donor-, recipient- and operative- related parameters. Donor parameters that are predictor of outcome are: advanced age, high body mass index (BMI), cause 
Table 2: Early (within 6 months) and late (after 6 months) complications after liver transplantation and their treatments

\begin{tabular}{|c|c|c|}
\hline Early & Diagnosis & Treatment \\
\hline Hepatic artery thrombosis & ultrasonography & thrombectomy, re-transplantation \\
\hline Portal vein thrombosis & ultrasonography & thrombectomy \\
\hline Hepatic vein thrombosis & ultrasonography & thrombectomy \\
\hline Bile leak & exam, US & percutaneous drainage, ERCP/stent \\
\hline Biliary stricture & lab, US & $\mathrm{ERCP} /$ stent, surgery \\
\hline Rejection & $\mathrm{Bx}$ & immunosuppression \\
\hline Infections & cultures & antibiotics \\
\hline Intra-abdominal abscess & $\mathrm{CT}$ & drainage, antibiotics \\
\hline \multicolumn{3}{|l|}{ Late } \\
\hline Hepatic artery stenosis & ultrasonography & endovascular, stent \\
\hline Portal vein stenosis & ultrasonography & endovascular, stent \\
\hline Biliary stricture & lab, US & ERCP/stent, surgery \\
\hline Chronic rejection & bx & re-transplantation \\
\hline Renal failure & lab & decrease tacrolimus, change to mTor \\
\hline Arterial hypertension & exam & medical treatment, decrease steroids \\
\hline Diabetes mellitus & lab & medical treatment, decrease steroids \\
\hline Dyslipidemia & lab & medical treatment, decrease steroids \\
\hline Metabolic syndrome & exam, lab & medical treatment, decrease steroids \\
\hline Bone complications & imaging & medical treatment, decrease steroids \\
\hline Neurological complications & exam, imaging & medical treatment, decrease tacrolimus \\
\hline Malignancy & exam, imaging & oncologic treatment, decrease immunosuppression \\
\hline Recurrent disease & exam, lab & medical treatment, re-transplantation \\
\hline Opportunistic infection & lab, cultures & antiviral or antifungal, decrease immnosuppresion \\
\hline $\begin{array}{l}\text { US: ultrasound } \\
\text { Bx: biopsy } \\
\text { ERCP: endoscopic retrograde cholnagiopancreatograpgy } \\
\text { mTOR: mammalian target of rapamycin }\end{array}$ & & \\
\hline
\end{tabular}

of brain death (stroke vs trauma), length of hospitalization, use of pressers, liver function, sodium level, reduced/split grafts vs whole graft, steatosis, and cold ischemia time. The recipient parameters include urgent status, renal dysfunction, age, ventilation requirement, and HCV. Operative factors are: the amount of blood loss and blood product administration, the lack of immediate bile production, low urine output, cold ischemic time $>12$ hours, and warm ischemia time $>45 \mathrm{~min}$. Finally, postoperative indicators are parameters such as elevated transaminase, serum bilirubin, serum creatinine, and prothrombin time. Liver transplant survival has increased over the past decade. One-year allograft and patients survival are approximately $85 \%$ and $90 \%$ respectively. Five-year allograft and patients survival are approximately $75 \%$ and $80 \%$ respectively. ${ }^{3}$

\section{ETHICAL APPROVAL}

There is nothing to be declared.

\section{CONFLICT OF INTEREST}

The author declares no conflict of interest related to this work.

\section{REFERENCES}

1. Malek-hosseini SA, Salahi H, Lahsaee M, Bahador A, Lankarani MB, Imanieh MH, et al. Initial experience with liver transplantation in Iran. Transplant Proc 2003;35:375-6. doi: 10.1016/S0041-1345(02)03844-7.

2. Jafarian A, Nassiri-Toosi M, Najafi A, Salimi J, Moini M, Azmoudeh-Ardalan F, et al. Establishing a liver transplantation program at Tehran University of Medical Sciences, Iran: a report of ten years of experience. Arch Iran Med 2014;17:81-3. doi: 0141701/AIM.0012. 
3. Saidi RF. Current Status of Liver Transplantation. Arch Iran Med 2012;15:772-6. doi: 0121512/ AIM.0011.

4. Ganji A, Malekzadeh F, Safavi M, Nasseri Moghaddam S, Nouraie M, Merat S, et.al. Digestive and Liver Disease Statistics in Iran. Middle East J Dig Dis 2009;1:56-62.

5. Stanaway JD, Flaxman AD, Naghavi M, Fitzmaurice C, Vos T, Abubakr I, et al. The global burden of viral hepatitis from 1990 to 2013: findings from the Global Burden of Disease Study 2013. Lancet 2016;388:10818. doi: 10.1016/S0140-6736(16)30579-7.

6. Malekzadeh F, Sepanlou SG, Poustchi H, Naghavi M, Forouzanfar MH, Shahraz S, et al. Burden of Gastrointestinal and Liver Diseases in Iran: Estimates Based on the Global Burden of Disease, Injuries, and Risk Factors Study, 2010. Middle East J Dig Dis 2015;7:138-54.

7. Sepanlou SG, Malekzadeh F, Naghavi M, Forouzanfar MH, Shahraz S, Moradi-Lakeh M, et al. Trend of Gastrointestinal and Liver Diseases in Iran: Results of the Global Burden of Disease Study, 2010. Middle East J Dig Dis 2015;7:121-37.

8. Malekzadeh MM, Vahedi H, Gohari K, Mehdipour P, Sepanlou SG, Ebrahimi Daryani N, et al. Emerging Epidemic of Inflammatory Bowel Disease in a Middle Income Country: A Nation-wide Study from Iran. Arch Iran Med 2016;19:2-15. doi: 0161901/ AIM.003.

9. Hajarizadeh B, Razavi-Shearer D, Merat S, Alavian SM, Malekzadeh R, Razavi H. Liver Disease Burden of Hepatitis $C$ Virus Infection in Iran and the Potential Impact of Various Treatment Strategies on the Disease Burden. Hepat Mon 2016;16:e37234. doi: 10.5812/hepatmon.37234.

10. Najafizadeh K, Abbasi A, Ghorbani F, Radpei B, Kashani BS, Ahmadi ZH, et al. Organ retrieval from brain-dead patients by a single organ procurement center in Iran. Transplant Proc 2009; 41:27235. doi: 10.1016/j.transproceed.2009.06.145.

11. Saidi RF, Hejazi Kenari SK. Liver Transplantation for Hepatocellular Carcinoma. Middle East J Dig Dis 2013;5:181-92.

12. Zimmerman A, Flahivr JM, Hertl M, Cosimi AB, Saidi RF. Outcomes of Full-Right-Full Left Split Liver Transplantation in Adults in the USA: A Propensity-Score Matched Analysis. Int J Organ Transplantat Med 2016;7:69-76.

13. Saidi RF. Utilization of Expanded Critieria Donors in Liver Transplantation. Int J Organ Transplantat Med 2013;4:46-59.

14. Saidi RF, Elias N, Ko DS, Kawai T, Markmann J, Feng S, et al. Live donor partial hepatectomy for liver transplantation: Is there a learning curve? Int $J$ Organ Transplantat Med 2010;1:125-30.
15. Saidi RF, Kenari SK. Liver Ischemia/Reperfusion Injury; an overview. J Invest Surg 2014;27:366-79. doi: 10.3109/08941939.2014.932473.

16. Hejazi Kenari K, Zimmerman A, Eslami M, Saidi RF. Current State of Art Management for Vascular Complications after Liver Transplantation. Middle East J Dig Dis 2014;6:121-30. 